\title{
SOME GENERAL RESULTS IN THE KINEMATICS OF AXISYMMETRICAL DEFORMATION OF SHELLS OF REVOLUTION*
}

\author{
BY \\ P. M. NAGHDI AND L. VONGSARNPIGOON \\ University of California, Berkeley
}

\begin{abstract}
This paper deals with some kinematical aspects of shells of revolution whose torsionless axisymmetrical deformation may be finite or one with small strain accompanied by large or moderately large rotation. The results are summarized in the form of three theorems.
\end{abstract}

1. Preliminary remark. The purpose of this paper is to state and prove certain purely kinematical results for torsionless axisymmetrical deformation of shells of revolution. The deformation of the shell may be finite or one with small strain accompanied by large or moderately large rotation. Since the results obtained are purely kinematical, they hold for all materials and are not necessarily limited to elastic shells. We utilize a direct formulation of the theory of shells based on a 2-dimensional continuum model as a Cosserat surface with a single director [1,2], but the corresponding kinematical developments can be also effected from an appropriate approximation for the position vector in the 3-dimensional theory (see, e.g., [2, Eq. (2.25)]).

For readers' convenience, we recall that a Cosserat surface is a body $\mathscr{C}$ comprising a material surface $\mathscr{S}$ embedded in a Euclidean 3 -space, together with a single deformable vector field-called director-attached to every material point of $\mathscr{S}$. The director, which is not necessarily along the unit normal to the surface surface $\mathscr{S}$, has in particular the property that it remains unaltered in length under superposed rigid body motions. ${ }^{1}$ Clearly, a Cosserat surface is not just a 2-dimensional surface; but is, in fact, endowed with some structure in the form of an additional primitive kinematical vector field. The magnitude of the director along the normals to the reference surface may be regarded as

\footnotetext{
${ }^{*}$ Received June 21,1983 . The results reported here we obtained in the course of research supported by the U.S. Office of Naval Research under Contract N00014-75-C-0148, Project NR 064-436 with the University of California, Berkeley.

${ }^{1}$ As in the paper of Naghdi [2], the director here is chosen to have the physical dimension of length. This differs from an earlier choice in Naghdi [1], where the director was assumed to be dimensionless. For further discussion on this point, see Naghdi [2, Sec. 3].
} 
representing the thickness of the shell-like body $\mathscr{B}$ and similarly the material surface $\mathscr{S}$ of $\mathscr{C}$ may be identified with the reference surface (e.g., the middle surface) in the shell-like body. Related remarks pertaining to the identification of various constitutive results, the assigned fields and the inertia coefficients in the theory of a Cosserat surface are made in Naghdi [1] and [2, Sec. 5]. Also, following the procedure of Casey and Naghdi [3], in the kinematical development of a Cosserat surface we remove the local rotation and the relative displacement at one point of the surface $\mathscr{S}$ resulting in a configuration $\kappa^{*}$ so as to render all kinematical and associated kinetical results invariant under arbitrary finite superposed rigid body motion (for details, see Naghdi and Vongsarnpigoon [4, Sec. 4-5] and [5, Sec. 2]).

By way of additional background and in the context of a 3-dimensional theory, consider a relative displacement ${ }^{2} \mathbf{u}^{*}=\mathbf{r}^{*}-\mathbf{R}^{*}$ and the relative displacement gradient $\mathbf{H}^{*}=\mathbf{F}^{*}-$ $\mathbf{I}=\operatorname{Grad} \mathbf{u}^{*}$, where $\mathbf{r}^{*}$ and $\mathbf{R}^{*}$ are, respectively, the position vectors of a material point in the deformed and undeformed configurations, $\mathbf{F}^{*}$ is the deformation gradient tensor and $\mathbf{I}$ is the identity tensor. Recall that through the polar decomposition theorem, we have $\mathbf{F}^{*}=\mathbf{R}^{*} \mathbf{U}^{*}$, where $\mathbf{R}^{*}$ is the (local) rotation tensor and $\mathbf{U}^{*}$ is the right stretch tensor which are related to the relative strain tensor $\mathbf{E}^{*}$ through the expression $2 \mathbf{E}^{*}=\left(\mathbf{U}^{*}\right)^{2}-\mathbf{I}$ $=\mathbf{F}^{* T} \mathbf{F}^{*}-\mathbf{I}$. If $\mathbf{H}^{*}$ is infinitesimal, then the strain $\mathbf{E}^{*}$ and the rotation $\mathbf{R}^{*}$ are also infinitesimal. But if only $\mathbf{E}^{*}$ is infinitesimal of order $\varepsilon$ the relative deformation $\mathbf{u}^{*}$ is not necessarily small and the rotation tensor $\mathbf{R}^{*}$ may be large or moderately large of order $\varepsilon^{1 / 2}$ (see Naghdi and Vongsarnpigoon [4]). Keeping the above background in mind, consider now a motion of a Cosserat surface $\mathscr{C}$ characterized by the two functions $\mathbf{r}$ and $\mathbf{d}$ corresponding to the position vector of a material point on the material surface $\mathscr{S}$ and the director at taht point. The deformation graident $\mathbf{F}$ and director gradient $\mathbf{G}$ can be defined in terms of suitable components of Grad $\mathbf{r}$ and Grad d (see Eqs. (A1) $)_{1,2}$ of Appendix A) and these, in turn, give rise to the strain tensor $2 \mathbf{E}=\mathbf{F}^{T} \mathbf{F}-\mathbf{I}$ and the curvature tensor $\mathbf{F}^{T} \mathbf{G}$ defined on the material surface $\mathscr{S}$ in the current configuration. Further, through the polar decomposition theorem, we again have $\mathbf{F}=\mathbf{R} \mathbf{U}$, where $\mathbf{R}$ is the (local) rotation tensor and $\mathbf{U}$ the right stretch tensor. Given $\mathbf{F}$, the position vector $\mathbf{r}$ and the director $\mathbf{d}$ in the deformed configuration $\kappa$ can be determined (see $\left.(\mathrm{A} 1)_{1}\right)$ and thus the deformation is known. A deformation of a shell-like body is said to be infinitesimal if the deformation gradient $\mathbf{F}$ differs only infinitesimally from the unit tensor $\mathbf{I}$ and, consequently, the strain tensor $\mathbf{E}$ and rotation tensor $\mathbf{R}$ are both infinitesimal. However, if $\mathbf{E}$ is small, the displacement vector $\mathbf{u}=\mathbf{r}-\mathbf{r}_{R}$, where $\mathbf{r}_{\mathbf{R}}$ is the position vector in the reference configuration, is not necessarily infinitesimal; and the deformation gradient tensor $\mathbf{F}$ could differ significantly from $\mathbf{I}$ and may be accompanied by large or moderately large $\mathbf{R}$.

Our main results are summarized as three theorems in Sec. 2 and the proofs are supplied in Sec. 3. Briefly, with reference to torsionless axisymmetrical deformation of a shell of revolution, we first show in Theorem 1 that the knowledge of the relative strain tensor at every point of the reference surface (e.g., the middle surface) is sufficient to

\footnotetext{
2 We attach an asterisk to the kinematical quantities associated with the 3-dimensional theory to differentiate them from corresponding quantities employed in the direct (2-dimensional) theory of a Cosserat surface.
} 
determine the change in the second fundamental form and hence the deformed configuration of the shell. Furthermore, if the strain and its first and higher order gradients are assumed to be small, then by our Theorem 2 the only kind of deformation accompanied by large rotation (or large deflection) - excluding, of course, the rigid body translation - of a nonshallow shell is that which involves an inversion of the shell. Indeed, it is a consequence of Theorem 2 that if the possibility of an inversion of the (nonshallow) shell is not admitted, a deformation with small strain accompanied by large rotation can take place only by allowing the strain gradient to be large. On the other hand, for a shallow shell of revolution (which includes an initially flat circular plate as a special case), while a deformation with large rotation (or large deflection) is not possible when the strain and strain gradients are assumed to be small, according to Theorem 3 the case in which the shell is subjected to a deformation with moderate rotation is admissible.

Throughout the paper, we use the usual summation convention over repeated indices with Greek indices taking the values 1, 2 and Latin indices having the ranges 1, 2, 3. Also, in the main text and the Appendix A, we employ both a coordinate-free notation and the component form of various results and equations.

2. Some background information. A summary of the main results. Let $\mathbf{e}_{i}(i=1,2,3)$ be a set of orthonormal base vectors associated with a system of rectangular Cartesian coordinates $(x, y, z)$ and let $\theta^{\alpha}(\alpha=1,2)$ designate a convected coordinate system on the material surface $\mathscr{S}$ of a Cosserat surface $\mathscr{C}$. Further, with reference to a cyclindrical polar coordinate system $(r, \theta, z)$, let $\mathbf{e}_{r}=\mathbf{e}_{r}(\theta)$ and $\mathbf{e}_{\theta}=\mathbf{e}_{\theta}(\theta)$ be the unit base vectors defined by

$$
\mathbf{e}_{r}=\cos \theta \mathbf{e}_{1}+\sin \theta \mathbf{e}_{2}, \quad \mathbf{e}_{\theta}=-\sin \theta \mathbf{e}_{1}+\cos \theta \mathbf{e}_{2} .
$$

Without loss in generality we may identify the convected coordinate $\theta^{2}$ with the angle $\theta$ of the cyclindrical polar coordinate system and write

$$
\theta^{2}=\theta
$$

Let $\mathscr{S}_{R}$, a two-dimensional region of space occupied by the material surface $\mathscr{S}$ of $\mathscr{C}$ in the reference configuration $\kappa_{0}$, be a surface of revolution with its position vector specified by

$$
\mathbf{r}_{R}=r_{0}\left(\theta^{1}\right) \mathbf{e}_{r}+z_{0}\left(\theta^{1}\right) \mathbf{e}_{3} \text {. }
$$

Then, from (2.3), the surface base vectors $\mathbf{A}_{\alpha}$ associated with the convected coordinates $\theta^{\alpha}$ and the outward unit normal $\mathbf{A}_{3}$ to $\mathscr{S}_{R}$ are calculated to be

$$
\mathbf{A}_{1}=r_{0}^{\prime} \mathbf{e}_{r}+z_{0}^{\prime} \mathbf{e}_{3}, \quad \mathbf{A}_{2}=r_{0} \mathbf{e}_{\theta}, \quad \mathbf{A}_{3}=\left(r_{0}^{\prime} \mathbf{e}_{3}-z_{0}^{\prime} \mathbf{e}_{r}\right) / \alpha_{0},
$$

where prime denotes partial differentiation with respect to $\theta^{1}$ and where $\alpha_{0}=\hat{\alpha}_{0}\left(\theta^{1}\right)$ is defined by

$$
\alpha_{0}^{2}=\left(r_{0}^{\prime}\right)^{2}+\left(z_{0}^{\prime}\right)^{2}
$$

The coefficients of the first and second fundamental forms of the material surface $\mathscr{S}$ in the reference configuration are

$$
\begin{gathered}
A_{11}=\alpha_{0}^{2}, \quad A_{22}=r_{0}^{2}, \quad A_{12}=0 \\
B_{11}=\left(r_{0}^{\prime} z_{0}^{\prime \prime}-z_{0}^{\prime} r_{0}^{\prime \prime}\right) / \alpha_{0}, \quad B_{22}=r_{0} z_{0}^{\prime} / \alpha_{0}, B_{12}=0 .
\end{gathered}
$$


Let $\mathbf{D}$ denote the director at $\mathbf{r}_{R}$ in the reference configuration $\boldsymbol{\kappa}_{0}$ of the Cosserat surface $\mathscr{C}$ and, for covenience, introduce the notation

$$
\mathbf{D}_{\alpha}=\mathbf{A}_{\alpha}, \quad \mathbf{D}_{3}=\mathbf{D} \text {. }
$$

In what follows, whenever desirable, the notations $\mathbf{D}_{i}=\left(\mathbf{D}_{1}, \mathbf{D}_{2}, \mathbf{D}_{3}\right)$ and the set (2.7), i.e., $\left(\mathbf{A}_{\alpha}, \mathbf{D}\right)$, will be used interchangeably depending on the particular context. Since $\mathbf{D}$ are linearly independent, as in Naghdi [2, Sec. 3], we may also introduce a set of reciprocal vectors $\mathbf{D}^{i}$ such that

$$
\mathbf{D}_{i} \cdot \mathbf{D}^{j}=\delta_{i}^{j}
$$

where $\delta_{i}^{j}$ is the Kronecker symbol in 3-space. Without loss in generality, we choose the director in the reference configuration to be along the normal to every point of $\mathscr{S}_{R}$ so that

$$
\mathbf{D}=D \mathbf{A}_{3}, \quad D=\hat{D}\left(\theta^{1}\right) .
$$

It follows from the choice (2.9) and the definition of $\mathbf{D}^{i}$ in (2.8) that

$$
\mathbf{D}^{\alpha}=\mathbf{A}^{\alpha}, \quad \mathbf{D}^{3}=\frac{1}{D} \mathbf{A}_{3},
$$

where $\mathbf{A}^{\alpha}$ are the surface reciprocal base vectors to $\mathbf{A}_{\alpha}$.

Let the Cosserat surface be deformed axisymmetrically without torsion such that the reference surface $\mathscr{S}_{R}$ becomes another surface of revolution with the position vector $\mathbf{r}=\hat{\mathbf{r}}\left(\theta^{\alpha}, t\right)$ in the current configuration $\boldsymbol{\kappa}$ specified by

$$
\mathbf{r}=r\left(\theta^{1}, t\right) \mathbf{e}_{r}+z\left(\theta^{1}, t\right) \mathbf{e}_{3} .
$$

Since the deformation is assumed to be torsionless, the meridians $\theta^{2}=$ const. and the parallels $\theta^{1}=$ const. of the undeformed surface become the meridians and the parallels, respectively, of the deformed surface. From (2.11), the surface base vector $\mathbf{a}_{\alpha}$ and the outward unit normal $\mathbf{a}_{3}$ are calculated to be

$$
\mathbf{a}_{1}=r^{\prime} \mathbf{e}_{r}+z \mathbf{e}_{3}, \quad \mathbf{a}_{2}=r \mathbf{e}_{\theta}, \quad \mathbf{a}_{3}=\left(-z^{\prime} \mathbf{e}_{r}+r^{\prime} \mathbf{e}_{3}\right) / \alpha .
$$

where the function $\alpha=\hat{\alpha}\left(\theta^{1}, t\right)$ is defined by

$$
\alpha^{2}=\left(r^{\prime}\right)^{2}+\left(z^{\prime}\right)^{2}
$$

The coefficients of the first and second fundamental forms in the deformed configuration $\kappa$ are given by ${ }^{3}$

$$
\begin{gathered}
a_{11}=\alpha^{2}, \quad a_{22}=r^{2}, \quad a_{12}=0, \\
b_{11}=\left(r^{\prime} z^{\prime \prime}-r^{\prime \prime} z^{\prime}\right) / \alpha, \quad b_{22}=r z^{\prime} / \alpha, \quad b_{12}=0 .
\end{gathered}
$$

Consistent with the assumption of torsionless axisymmetrical motion, similarly to $\mathbf{r}$ given by (2.11), the director $\mathbf{d}=\hat{\mathbf{d}}\left(\theta^{\alpha}, t\right)$ in the deformed configuration $\boldsymbol{\kappa}$ must also lie in the plane spanned by $\mathbf{a}_{1}$ and $\mathbf{a}_{3}$ so that

$$
\mathbf{d} \cdot \mathbf{a}_{2}=0
$$

\footnotetext{
${ }^{3}$ As noted in Sec. 1 (at the end of the second paragraph), the deformed configuration here is actually a configuration $\kappa^{*}$ but we have suppressed the asterisk.
} 
everywhere, and the components of $\mathbf{d}$ along $\mathbf{a}_{1}$ and $\mathbf{a}_{3}$ are independent of $\boldsymbol{\theta}$. Again, for convenience, we introduce the notation

$$
\mathbf{d}_{\alpha}=\mathbf{a}_{\alpha}, \quad \mathbf{d}_{3}=\mathbf{d},
$$

and denote by $\mathbf{d}^{i}$ the reciprocal base vectors to $\mathbf{d}_{i}$ such that $\mathbf{d}_{i} \cdot \mathbf{d}^{j}=\delta_{i}^{j}$.

As indicated in Appendix $\mathrm{A}$, the relative strain tensor $\mathbf{E}=\hat{\mathbf{E}}\left(\theta^{\alpha}, t\right)$ and the relative curvature tensor $\mathbf{J}=\hat{\mathbf{J}}\left(\theta^{\alpha}, t\right)$ defined by Eqs. (A2) $)_{1,2}$ can be expressed as

$$
\mathbf{E}=\gamma_{i j} \mathbf{D}^{i} \otimes \mathbf{D}^{j}, \quad \mathbf{J}=\kappa_{i \alpha} \mathbf{D}^{i} \otimes \mathbf{D}^{\alpha},
$$

and their components $\gamma_{i j}$ and $\kappa_{i \alpha}$ are given by

$$
\begin{aligned}
& \gamma_{11}=\frac{1}{2}\left(\alpha^{2}-\alpha_{0}^{2}\right), \quad \gamma_{22}=\frac{1}{2}\left(r^{2}-r_{0}^{2}\right), \gamma_{12}=0, \\
& \gamma_{13}=\frac{1}{2} \mathbf{d} \cdot \mathbf{a}_{1}, \quad \gamma_{33}=\frac{1}{2}\left(\mathbf{d} \cdot \mathbf{d}-D^{2}\right), \gamma_{23}=0,
\end{aligned}
$$

and

$$
\kappa_{\beta \alpha}=\lambda_{\beta \alpha}-\left(-D B_{\beta \alpha}\right), \quad \lambda_{\beta \alpha}=\mathbf{d}_{\beta} \cdot \mathbf{d}_{, \alpha}, \quad \kappa_{3 \alpha}=\gamma_{33, \alpha} .
$$

We also recall the definition of the change in the second fundamental form $\beta_{\alpha \beta}=b_{\alpha \beta}-B_{\alpha \beta}$ and calculate from (2.6) and (2.14) the expressions

$$
\beta_{11}=\frac{\left(r^{\prime} z^{\prime \prime}-r^{\prime \prime} z^{\prime}\right)}{\alpha}-\frac{\left(r_{0}^{\prime} z_{0}^{\prime \prime}-r_{0}^{\prime \prime} z_{0}^{\prime}\right)}{\alpha_{0}}, \quad \beta_{22}=\frac{r z^{\prime}}{\alpha}-\frac{r_{0} z_{0}^{\prime}}{\alpha_{0}}, \quad \beta_{12}=0 .
$$

In what follows, when the deformation is such that the strainis small, we write

$$
\mathbf{E}=\mathbf{O}(\varepsilon) \text {, }
$$

where $\mathbf{O}$ denotes the usual order of magnitude symbol, i.e., a function $f$ is said to be of $O\left(\varepsilon^{n}\right)$ if there exists a constant $C$ such that $\|f\|<C \varepsilon^{n}$ as $\varepsilon \rightarrow 0$, where the notation $\|f\|$ stands for the norm or magnitude of $f$. We note that (2.21) implies that the components $\gamma_{i j}$ of $\mathbf{E}$ are also of $O(\varepsilon)$ but this does not imply that the rotation $\mathbf{R}$ or the relative displacement $\mathbf{u}$ are small.

Certain features of steep (nonshallow) and shallow shells have been noted previously in [5]. Here, with reference to shells of revolution, it is more convenient to stipu.ate a measure of shallowness in terms of the rise $z_{0}$ of the reference surface (e.g., the middle surface) of the shell of revolution. Assuming that $z_{0}$ does not vary rapdily (as in the case of a corrugated shell), i.e., the slope $z_{0}^{\prime}$ is not of greater order of magnitude than $z_{0}$, then it can be easily seen that a steep shell of revolution can be defined by

$$
z_{0}^{\prime}=O(1)
$$

and a shallow shell of revolution may be defined by

$$
z_{0}^{\prime}=O\left(\varepsilon^{1 / 2}\right) \text {. }
$$

The latter, along with the expressions $\left(2.6_{4,5,6}\right)$, imply that $B_{\alpha \beta}=0\left(\varepsilon^{1 / 2}\right)$ which is consistent with that used in [5, Eq. (4.7)].

We now state three theorems applicable to torsionless, axisymmetrical deformations of a shell of revolution. The first theorem concerns a general deformation with no restriction on the strain or the rotation, but the next two concern a deformation with infinitesimal strain. 
THEOREM 1. The surface components $\gamma_{\alpha \beta}$ of the strain $\gamma_{i j}$ in (2.18) determine the coefficients of the second fundamental form in (2.14) of the deformed configuration, as well as the change of the second fundamental form given by (2.20). Furthermore, the deformation of the shell-like body (including the local rotation $\mathbf{R}$ ) is then determined.

THEOREM 2. For a deforming shell which is nonshallow in the reference state as specified by (2.22), if the components of the strain $\gamma_{i j}$ and its first and higher order gradients are all infinitesimal of $O(\varepsilon)$, then the deformation is either infinitesimal or one with small strain accompanied by large rotation. The latter necessarily involves an inversion of the shell.

THEOREM 3. For a shallow shell of revolution, if the components of the strain $\gamma_{i j}$ and the first and higher order gradients of the strain are infinitesimal of $O(\varepsilon)$, then the deformation is at most one with small strain of $O(\varepsilon)$ accompanied by moderate rotation of $O\left(\varepsilon^{1 / 2}\right)$.

Suplementary to Theorem 2 , the following remark may be noted:

Remark 1. If only a part of the nonshallow shell of revolution is inverted while the rest is not, the two regions should be considered separately because $z^{\prime}$ is discontinuous at the interface of the two regions.

The following corollary is an immediate consequence of Theorem 3 in the special case in which the surface $\mathscr{S}_{R}$ is a plane so that $\mathscr{C}$ can be regarded as representing a circular plate in its reference configuration:

Corollary 1 . If the surface $\mathscr{S}_{R}$ is a plane and $\mathscr{C}$ is subjected to a torsionless axisymmetrical deformation for which the components of the strain $\gamma_{i j}$ and the first and higher gradients of strain all being of $O(\varepsilon)$, then the deformation is at most one with small strain of $O(\varepsilon)$ accompanied by moderate rotation of $O\left(\varepsilon^{1 / 2}\right)$.

It is well known in differential geometry that given the coefficients of the first and second fundamental forms, the surface is determined to within a rigid body motion (see, for example, Eisenhart [6, p. 221] or Theorem 8.3 in Ch. 6 of O'Neill [7]). However, in the context of axisymmetrical deformation of shells of revolution, it is evident from Theorem 1 that the knowledge of the coefficients of the first fundamental form is sufficient to determine the surface (including the coefficients of the second fundamental form). It is therefore of some interest to ask if an inverse to the Theorem 1 exists in the sense that does the knowledge of the second fundamental form determine the deformation functions and hence also the first fundamental form of the surface? In order to examine this question, assume that the components $b_{11}=f_{1}\left(\theta^{1}, t\right)$ and $b_{22}=f_{2}\left(\theta^{1}, t\right)$ are known, then in principle the functions $r\left(\theta^{1}, t\right)$ and $z\left(\theta^{1}, t\right)$ can be determined from the differential equations

$$
\frac{r^{\prime} z^{\prime \prime}-r^{\prime \prime} z^{\prime}}{\left[\left(r^{\prime}\right)^{2}+\left(z^{\prime}\right)^{2}\right]^{1 / 2}}=f_{1}, \quad \frac{r z^{\prime}}{\left[\left(r^{\prime}\right)^{2}+\left(z^{\prime}\right)^{2}\right]^{1 / 2}}=f_{2},
$$

subject to appropriate boundary conditions. Once $r$ and $z$ are known, the components $\gamma_{\alpha \beta}$ of the strain can be easily calculated. Alternatively, given the Gaussian curvature $K=$ $K\left(\theta^{1}, t\right)$ and the mean curvature $H=H\left(\theta^{1}, t\right)$, we can write the following differential 
equations for $r\left(\theta^{1}, t\right)$ and $z\left(\theta^{1}, t\right)$, namely ${ }^{4}$

$$
\begin{gathered}
\frac{r^{\prime} z^{\prime} z^{\prime \prime}-r^{\prime \prime}\left(z^{\prime}\right)^{2}}{r\left[\left(r^{\prime}\right)^{2}+\left(z^{\prime}\right)^{2}\right]^{2}}=K, \\
\frac{r\left(r^{\prime} z^{\prime \prime}-r^{\prime \prime} z^{\prime}\right)+z^{\prime}\left[\left(r^{\prime}\right)^{2}+\left(z^{\prime}\right)^{2}\right]}{r\left[\left(r^{\prime}\right)^{2}+\left(z^{\prime}\right)^{2}\right]^{3 / 2}}=2 H .
\end{gathered}
$$

It follows from elementary results in differential geometry that the shape (but not the strain measures) of a surface of revolution is known once $H$ and $K$ are known (see Lemma 6.3 in Ch. 5 of O'Neill [7]). For example, if $H=0$, then the surface of revolution is either a plane or a catenoid (see, e.g., Theorem 6.2 in Ch. 5 of O'Neill [7]). Several examples of surfaces with constant Gaussian curvature are also given in O'Neill ([7], Ch. 5, Sec. 6). These known results may allow the specification of the functions $r$ and $z$ in some cases without actually solving (2.24) or (2.25). However, in general, there may be more than one set of deformation functions $r\left(\theta^{1}, t\right)$ and $z\left(\theta^{1}, t\right)$ which satisfy (2.24) or (2.25). For example, if $f_{1}=f_{2}=0$, then the deformed surface is a circular plane, $z=$ constant and the equations $(2.24)_{1,2}$ are now identically satisfied without any restriction on the function $r\left(\theta^{1}, t\right)$. In fact, $r$ can be any arbitrary function of $\theta^{1}$ and $t$ which does not violate the conditions imposed at the edge of the circular plane, or its center point.

The foregoing theorems for axisymmetrical deformation of shells of revolution after appropriate specialization are applicable also to various special cases of the nonlinear theory of shells obtained either by direct approach or a corresponding development for the 3-dimensional equations. Among these, mention may be made of the constrained theory discussed in [2, Sec. 6] or a restricted theory in [1, Sec. 10] which is constructed by direct approach and which corresponds to the Kirchoff-Love theory of shells in the presence of finite deformation [8] or a development such as that discussed by Reissner [9].

Before closing this section, we make some remarks pertaining to Reissner's [9] analysis of axisymmetric deformation of shells of revolution in the light of the above three theorems. In Reissner's [9] discussion of axisymmetrical deformation of shells of revolution, both finite and "small finite" deflections are allowed. Although the strain in Reissner's paper is assumed to be small, no restriction is imposed on the strain gradient. In the light of Theorem 2, if the strain gradient is small and if the inversion of the shell is ruled out, it is not possible to have a finite deflection of a steep shell of revolution.

Thus, in allowing for finite deflection in Reissner's work [9], one must assume that the strain gradients are finite unless an inversion of the shell is allowed. Similarly, if a "small finite" deflection of a nonshallow shell in the sense of Reissner [9, Sec. 4] is allowed, then again this is not possible unless one also assumes that the strain gradient is moderate of $O\left(\varepsilon^{1 / 2}\right)$ as characterized in Naghdi and Vongsarnipgoon [5]. Consequently, with the use of linear constitutive relations (with constant coefficients), the gradients of the resultant forces must be viewed as finite or moderate. In addition, since the strain gradient may be large, care must be exercised when the equations of motion are simplified under the

\footnotetext{
${ }^{4}$ Recall that the principal curvatures $b_{1}^{1}$ and $b_{2}^{2}$ can be easily determined from the knowledge of $H$ and $K$.
} 
assumption of small strain. Specifically, it appears that Reissner [9] intends for his equations of equilibrium (1)' to be valid for both finite and "small finite" deformation. In view of Theorem 2, it can be argued that equations (1)' and associated results are valid for "small finite" deformation but these may not yield reliable results for the case of finite deflection. To elaborate further, recall that Reissner [9] obtains his set of equations (1)' by replacing the variables $r$ and $\alpha$ by their values $r_{0}$ and $\alpha_{0}$ in the reference configuration. However, this procedure is incorrect if the strain gradient is large. Consider, for example, the first term on the left-hand side of Reissner's Eq. $(1)_{2}$ which upon expansion yields (in Reissner's notation)

$$
\begin{aligned}
(r H)^{\prime} & =\left[\left(1+\varepsilon_{\theta M}\right) r_{0} H\right]^{\prime} \\
& =\left(1+\varepsilon_{\theta M}\right)\left(r_{0} H\right)^{\prime}+\varepsilon_{\theta M}^{\prime}\left(r_{0} H\right) \\
& \approx\left(r_{0} H\right)^{\prime}+\varepsilon_{\theta M}^{\prime}\left(r_{0} H\right) .
\end{aligned}
$$

Since $\varepsilon_{\theta M}^{\prime}$ must be finite for a finite deflection without inversion, the term $\varepsilon_{\theta M}^{\prime}\left(r_{0} H\right)$ is not negligible and should be retained in the modified equation. For a deformation with moderate rotation (or a "small finite" deflection), if terms of $O\left(\varepsilon^{1 / 2}\right)$ are considered small and negligible in comparison with ${ }^{5}$ unity, then $\varepsilon_{\theta M}^{\prime}\left(r_{0} H\right)$ is negligible and the set of equations (1)' can be used.

In the case of a shallow shell or plate, if the strain and strain gradients are infinitesimal, then the possibility of a finite deflection is ruled out in the light of Theorem 3. Thus, in order to consider finite deflection of an initially flat plate as in [9, Sec. 5], the strain gradient must be regarded as finite although this has not been explicitly stated in [9]. It should be noted that according to Theorem 3 , it is possible for a shallow shell or a plate to deform with moderate rotation while the strain and strain gradients remain infinitesimal; and, for a deformation of this kind, the use of an expansion of the type (14) in [9, Sec. 4] -intended for "small finite" deflection—is valid.

3. Proofs of the theorems. We provide here the details of the proofs of the theorems stated in the previous section. In order to prove Theorem. 1, we first note from (2.18) 1.2 that

$$
\alpha^{2}=\alpha_{0}^{2}+2 \gamma_{11}, \quad r^{2}=r_{0}^{2}+2 \gamma_{22} .
$$

Since $r$ is always positive, the square root of $(3.1)_{2}$ yields

$$
r=r_{0}\left(1+2 \gamma_{22} / r_{0}^{2}\right)^{1 / 2}
$$

Differentiaging (3.2) with respect to $\theta^{1}$, we have

$$
r^{\prime}=\left(r_{0} r_{0}^{\prime}+\gamma_{22}^{\prime}\right)\left(r_{0}^{2}+2 \gamma_{22}\right)^{-1 / 2}
$$

Next, with the help of (3.1) and (3.3), we may solve for $z^{\prime}$ from (2.13) to obtain

$$
z^{\prime}= \pm\left[\alpha^{2}-\left(r^{\prime}\right)^{2}\right]^{1 / 2}= \pm\left\{\alpha_{0}^{2}+2 \gamma_{11}-\left(r_{0} r_{0}^{\prime}+\gamma_{22}^{\prime}\right)^{2} /\left(r_{0}^{2}+2 \gamma_{22}\right)\right\}^{1 / 2} \text {. }
$$

\footnotetext{
${ }^{5}$ Alternatively, the same conclusion can be obtained by neglecting terms of $O\left(\varepsilon^{3 / 2}\right)$ in comparison with terms of $O(\varepsilon)$ as in the procedure of Naghdi and Vongsarnpigoon [5].
} 
It is clear from (3.4) that once the sign of $z^{\prime}$ has been chosen, the knowledge of $\gamma_{11}$ and $\gamma_{22}$ determine $z^{\prime}$. Since $\alpha, r, r^{\prime}, z^{\prime}$ and $r^{\prime \prime}, z^{\prime \prime}$ can be determined from the knowledge of $\gamma_{\alpha \beta}$, it follows from (2.14) $)_{4-6}$ and (2.20) $)_{1-3}$ that the coefficients of the second fundamental form $b_{\alpha \beta}$ and the change in the second fundamental form $\beta_{\alpha \beta}$ can be determined from $\gamma_{\alpha \beta}$. Furthermore, by (A4), (A5) and (A2) ${ }_{3}$ of Appendix A, $\lambda_{\alpha \beta}$ (and hence $\mathbf{F}^{T} \mathbf{G}$ ) are also determined once $b_{\alpha \beta}$ and $\gamma_{i j}$ are known. Since the deformation of the Cosserat surface $\mathscr{C}$ can be determined to within a rigid motion, and since as noted in Sec. 1 we have identified the deformation configuration with the configuration $\kappa^{*}$ of $\mathscr{C}$, i.e., the configuration in which the local rotation and the displacement have been removed from one point of the body, the deformation of $\mathscr{C}$ is determined and the proof is complete.

The sign of $z^{\prime}$ provides information about the manner in which the shell has deformed. If the sign of $z^{\prime}$ is opposite to that of $z_{0}^{\prime}$, the deformation involves an inversion of the shell. Also, it should be noted that in the special case in whcih the surface $\mathscr{S}_{R}$ of $\mathscr{C}$ is a plane, i.e., when $z_{0}^{\prime}=0$, the sign of $z^{\prime}$ will be the same as the sign of the component $b_{22}=\beta_{22}$ of the second fundamental form in the deformed configuration.

We now turn to theproof of Theorem 2. Keeping in mind that both the strain and its first and second gradients are of $O(\varepsilon)$, if terms of $O\left(\varepsilon^{2}\right)$ are neglected in $(2.18)_{1,2}$, we may write

$$
\alpha=\alpha_{0}+\frac{\gamma_{11}}{\alpha_{0}}=\alpha_{0}+O(\varepsilon), \quad r=r_{0}+\frac{\gamma_{22}}{r_{0}}=r_{0}+O(\varepsilon),
$$

and differentiation of $(3.5)_{2}$ yields

$$
r^{\prime}=r_{0}^{\prime}+\left(\frac{\gamma_{22}}{r_{0}}\right)^{\prime}=r_{0}^{\prime}+O(\varepsilon)
$$

Squaring both sides of (3.6) and neglecting terms of $O\left(\varepsilon^{2}\right)$ we have

$$
\left(r^{\prime}\right)^{2}=\left(r_{0}^{\prime}\right)^{2}+2 r_{0}^{\prime}\left(\frac{\gamma_{22}}{r_{0}}\right)^{\prime}=\left(r_{0}^{\prime}\right)^{2}+O(\varepsilon) .
$$

Then, making use of (3.5) 1 and (3.7), (2.13) can be rewritten as

$$
\left(z^{\prime}\right)^{2}=\alpha^{2}-\left(r^{\prime}\right)^{2}=\left(\alpha_{0}^{2}+2 \gamma_{11}\right)-\left(r_{0}^{\prime}\right)^{2}-2 r_{0}^{\prime}\left(\gamma_{22} / r_{0}\right)^{\prime}
$$

or

$$
\left(z^{\prime}\right)^{2}=\left(z_{0}^{\prime}\right)^{2}+2 \gamma_{11}-2 r_{0}^{\prime}\left(\gamma_{22} / r_{0}\right)^{\prime}=\left(z_{0}^{\prime}\right)^{2}+O(\varepsilon) .
$$

Recalling from (2.22) that $z_{0}^{\prime}=O(1)$ for a steep shell, the square root of $(3,8)$ after the neglect of terms of $O\left(\varepsilon^{2}\right)$ gives

$$
\begin{aligned}
z^{\prime} & = \pm\left(z_{0}^{\prime}\right)\left\{1+\frac{\gamma_{11}}{\left(z_{0}^{\prime}\right)^{2}}-\frac{r_{0}^{\prime}}{\left(z_{0}^{\prime}\right)^{2}}\left(\frac{\gamma_{22}}{r_{0}}\right)^{\prime}\right\} \\
& = \pm\left\{z_{0}^{\prime}+\frac{\gamma_{11}}{z_{0}^{\prime}}-\frac{r_{0}^{\prime}}{z_{0}^{\prime}}\left(\frac{\gamma_{22}}{r_{0}}\right)^{\prime}\right\} \\
& = \pm z_{0}^{\prime}+O(\varepsilon),
\end{aligned}
$$


and hence also

$$
z^{\prime \prime}= \pm\left\{z_{0}^{\prime \prime}+\left[\frac{\gamma_{11}}{z_{0}^{\prime}}\right]^{\prime}-\left[\frac{r_{0}^{\prime}}{z_{0}^{\prime}}\left(\frac{\gamma_{22}}{r_{0}}\right)^{\prime}\right]^{\prime}\right\}= \pm z_{0}^{\prime \prime}+O(\varepsilon)
$$

To proceed further, consider first the case in which the plus sign is chosen in (3.9) and (3.10), i.e., the case in which $z^{\prime}$ has the same sign as $z_{0}^{\prime}$ and there is no inversion of the shell. With the use of (3.9), (3.10), (3.6) and (3.5) $)_{1,2}$ in $(2.20)_{1,2}$ and after the neglect of terms of $O\left(\varepsilon^{2}\right)$, the nonvanishing components of $\beta_{\alpha \beta}$ can be written as

$$
\begin{aligned}
\beta_{11}= & \left\{z_{0}^{\prime \prime}\left(\frac{\gamma_{22}}{r_{0}}\right)^{\prime}+r_{0}^{\prime}\left[\frac{\gamma_{11}}{z_{0}^{\prime}}-\frac{r_{0}^{\prime}}{z_{0}^{\prime}}\left(\frac{\gamma_{22}}{r_{0}}\right)^{\prime}\right]^{\prime}-z_{0}^{\prime}\left(\frac{\gamma_{22}}{r_{0}}\right)^{\prime \prime}\right. \\
& \left.-r_{0}^{\prime \prime}\left[\frac{\gamma_{11}}{z_{0}^{\prime}}-\frac{r_{0}^{\prime}}{z_{0}^{\prime}}\left(\frac{\gamma_{22}}{r_{0}}\right)^{\prime}\right]-\frac{\gamma_{11}}{\alpha_{0}^{2}}\left(r_{0}^{\prime} z_{0}^{\prime \prime}-r_{0}^{\prime \prime} z_{0}^{\prime}\right)\right\} / \alpha_{0} \\
= & O(\varepsilon), \\
\beta_{22}= & \left\{r_{0} \frac{\gamma_{11}}{z_{0}^{\prime}}-r_{0} \frac{r_{0}^{\prime}}{z_{0}^{\prime}}\left(\frac{\gamma_{22}}{r_{0}}\right)^{\prime}+z_{0}^{\prime}\left(\frac{\gamma_{22}}{r_{0}}\right)-r_{0} z_{0}^{\prime} \frac{\gamma_{11}}{\alpha_{0}^{2}}\right\} / \alpha_{0}=O(\varepsilon) .
\end{aligned}
$$

Since $\beta_{\alpha \beta}=O(\varepsilon)$, by Theorem A3 of Appendix A the rotation tensor is an infinitesimal rotation with respect to $\varepsilon$ everywhere and the deformation is infinitesimal. On other hand, if the minus sign is chosen in (3.9) and (3.10), i.e., the case in which the deformation involves the inversion of the shell, then instead of $(3.11)_{1,2}$ we obtain

$$
\begin{aligned}
\beta_{11}= & -2 \frac{\left(r_{0}^{\prime} z_{0}^{\prime \prime}-r_{0}^{\prime \prime} z_{0}^{\prime}\right)}{\alpha_{0}}-\left\{z_{0}^{\prime \prime}\left(\frac{\gamma_{22}}{r_{0}}\right)^{\prime}+r_{0}^{\prime}\left[\frac{\gamma_{11}}{z_{0}^{\prime}}-\frac{r_{0}^{\prime}}{z_{0}^{\prime}}\left(\frac{\gamma_{22}}{r_{0}}\right)^{\prime}\right]^{\prime}-z_{0}^{\prime}\left(\frac{\gamma_{22}}{r_{0}}\right)^{\prime \prime}\right. \\
& \left.-r_{0}^{\prime \prime}\left[\frac{\gamma_{11}}{z_{0}^{\prime}}-\frac{r_{0}^{\prime}}{z_{0}^{\prime}}\left(\frac{\gamma_{22}}{r_{0}}\right)^{\prime}\right]-\frac{\gamma_{11}}{\alpha_{0}^{2}}\left(r_{0}^{\prime} z_{0}^{\prime \prime}-r_{0}^{\prime \prime} z_{0}^{\prime}\right)\right\} / \alpha_{0} \\
= & -2 B_{11}+O(\varepsilon), \\
B_{22}= & -2 \frac{r_{0} z_{0}^{\prime}}{\alpha_{0}}-\left\{r_{0} \frac{\gamma_{11}}{z_{0}}-r_{0} \frac{r_{0}^{\prime}}{z_{0}^{\prime}}\left(\frac{\gamma_{22}}{r_{0}}\right)^{\prime}+z_{0}^{\prime}\left(\frac{\gamma_{22}}{r_{0}}\right)-r_{0} z_{0}^{\prime} \frac{\gamma_{11}}{\alpha_{0}^{2}}\right\} / \alpha_{0} \\
= & -2 B_{22}+O(\varepsilon) .
\end{aligned}
$$

Since $z_{0}^{\prime}=O(1)$, we have $B_{\alpha \beta}=O(1)$ and it follows from (3.12) $)_{1,2}$ that $\beta_{\alpha \beta}=O(1)$ also. The rotation tensor in this case is finite by Theorem A1 of Appendix A and hence the deformation is also finite. This completes the proof of Theorem 2.

The conclusions in Theorem 2 involving the choice of sign in (3.19), i.e., the plus sign leading to infinitesimal deformation and the minus sign leading to finite deformation, can also be reached from direct integration of (3.9) subject to the condition that the displacement vector given by $\left(r-r_{0}\right) \mathbf{e}_{r}+\left(z-z_{0}\right) \mathbf{e}_{3}$ vanishes at one point of the material surface $\mathscr{S}$.

We consider next the proof of Theorem 3. First we note that the results (3.5)-(3.8) pertaining to Theorem 2 remain valid in this case also. However, since $z_{0}^{\prime}=O\left(\varepsilon^{1 / 2}\right)$ for 
shallow shells by $(2.23),\left(z_{0}^{\prime}\right)^{2}=O(\varepsilon)$ and hence by (3.8) we have

$$
\left(z^{\prime}\right)^{2}=O(\varepsilon)
$$

and its square root is

$$
\left(z^{\prime}\right)=O\left(\varepsilon^{1 / 2}\right) .
$$

Differentiating (3.8) with respect to $\theta^{1}$, we have

$$
z^{\prime} z^{\prime \prime}=z_{0}^{\prime} z_{0}^{\prime \prime}+\left[\gamma_{11}-r_{0}^{\prime}\left(\frac{\gamma_{22}}{r_{0}}\right)^{\prime}\right]^{\prime} .
$$

Since the right-hand side of (3.15) is of $O(\varepsilon)$, its left-hand side must also be of $O(\varepsilon)$ and with the use of (3.14), we conclude that

$$
z^{\prime \prime}=O\left(\varepsilon^{1 / 2}\right) .
$$

With the use of (3.6) and its derivative, as well as (3.14) and (3.16), it follows from (2.20) that

$$
\beta_{\alpha \beta}=O\left(\varepsilon^{1 / 2}\right)
$$

and by Theorem A2 of Appendix A, the rotation tensor is a moderate rotation with respect to $\varepsilon$. (See also Theorem 3.2 of Naghdi and Vongsarnpigoon [5].) for the special case in which the right-hand side of (3.13) is of $O\left(\varepsilon^{2}\right), z^{\prime}$ and $z^{\prime \prime}$ are of $O(\varepsilon), \beta_{\alpha \beta}=O(\varepsilon)$ and the deformation is infinitesimal. This completes the proof of Theorem 3 .

Consider now the proof of the statement in Remark 1 to the effect that $z^{\prime}$ is discontinuous at the interface between the inverted and noninverted regions of an initially steep shell. Suppose $z^{\prime}$ is continuous and varies smoothly from a value which has the same sign as $z_{0}^{\prime}$ to that of an opposite sign. Then, at some point in the region $z^{\prime}$ must vanish and (3.8) must hold with the left-hand side set equal to zero. Since $z_{0}^{\prime}$ is known and finite, the quantity $\left[2 \gamma_{11}-2 r_{0}^{\prime}\left(\gamma_{22} / r_{0}\right)^{\prime}\right]$ must also be finite to cancel $\left(z_{0}^{\prime}\right)^{2}$. However, by assumption, the strain and strain gradients are infinitesimal everywhere. Hence, $\left[2 \gamma_{11}-2 r_{0}^{\prime}\left(\gamma_{22} / r_{0}\right)^{\prime}\right]$ is of $O(\varepsilon)$ and we have a contradiction. This completes the proof of Remark 1.

Finally, since an initially flat plate can be regarded as a special case of a shallow shell with $B_{\alpha \beta}=0$ and $z_{0}^{\prime}=z_{0}^{\prime \prime}=0$, the Corollary 1 follows as a special case of Theorem 3. It should be noted that for an initially circular plate, $(3.8)$ reduces to

$$
\left(z^{\prime}\right)^{2}=2 \gamma_{11}-2 r_{0}^{\prime}\left(\frac{\gamma_{22}}{r_{0}}\right)^{\prime}=O(\varepsilon) .
$$

Appendix A. In this appendix, we consider some additional kinematical results for a shell of revolution. Recalling the definitions of the base vectors $\mathbf{d}_{i}$ and $\mathbf{D}_{i}$, the deformation gradient $\mathbf{F}=\hat{\mathbf{F}}\left(\theta^{\alpha}, t\right)$ and the director gradient $\mathbf{G}=\hat{\mathbf{G}}\left(\theta^{\alpha}, t\right)$ are defined by (see [2, Sec. 8]):

$$
\begin{aligned}
& \mathbf{F}=\mathbf{d}_{i} \otimes \mathbf{D}^{i}=\mathbf{a}_{\alpha} \otimes \mathbf{A}^{\alpha}+\frac{1}{D} \mathbf{D} \otimes \mathbf{A}_{3}, \\
& \mathbf{G}=\mathbf{d}_{3, \alpha} \otimes \mathbf{D}^{\alpha}=\mathbf{d}_{, \alpha} \otimes \mathbf{A}^{\alpha},
\end{aligned}
$$


where a comma denotes partial differentiation with respect to $\theta^{\alpha}$. The relative strain tensor $\mathbf{E}=\hat{\mathbf{E}}\left(\theta^{\alpha}, t\right)$ and the relative curvature tensor $\mathbf{J}=\hat{\mathbf{J}}\left(\theta^{\alpha}, t\right)$ are given by

$$
\mathbf{E}=\frac{1}{2}\left(\mathbf{F}^{T} \mathbf{F}-\mathbf{I}\right), \quad \mathbf{J}=\mathbf{F}^{T} \mathbf{G}-{ }_{0} \mathbf{G}, \quad \mathbf{F}^{T} \mathbf{G}=\lambda_{i \alpha} \mathbf{D}^{i} \otimes \mathbf{D}^{\alpha},
$$

where ${ }_{0} \mathbf{G}$ is the reference value of $\mathbf{G}$ ( or $\left.\mathbf{F}^{T} \mathbf{G}\right)$, i.e.,

$$
{ }_{0} \mathbf{G}=\mathbf{D}_{, \alpha} \otimes \mathbf{A}^{\alpha}=-D B_{\beta \alpha} \mathbf{A}^{\beta} \otimes \mathbf{A}^{\alpha}+D_{, \alpha} \mathbf{A}_{3} \otimes \mathbf{A}^{\alpha} .
$$

The components of $\mathbf{E}$ and $\mathbf{J}$ in (2.17)-(2.19) can be obtained after substituting the expressions for $\mathbf{F}$ and $\mathbf{G}$ from (A1) into (A2) and making use of (2.4), (2.9), (2.12), (2.14) and (2.15). We also note that

$$
\begin{aligned}
& \mathbf{d} \cdot \mathbf{a}_{\alpha}=\mathbf{d}_{3} \cdot \mathbf{d}_{\alpha}=2 \gamma_{3 \alpha}, \\
& \mathbf{d} \cdot \mathbf{d}=D^{2}+2 \gamma_{33}=\left(\mathbf{d} \cdot \mathbf{a}_{3}\right)^{2}+a^{\alpha \beta} \gamma_{3 \alpha} \gamma_{3 \beta},
\end{aligned}
$$

where $a^{\alpha \beta}$ is the conjugate surface metric of the deformed surface. By straightforward calculation, it can be seen that $\lambda_{\beta \alpha}$ are related to $b_{\alpha \beta}$ by

$$
\lambda_{\beta \alpha}=\gamma_{3 \beta \mid \alpha}-b_{\beta \alpha}\left(\mathbf{d} \cdot \mathbf{a}_{3}\right),
$$

where a bar denotes covariant differentiation with respect to the metric $a_{\alpha \beta}$. The relationship between $\kappa_{\beta \alpha}$ and $\beta_{\alpha \beta}$ is clear from (A5).

Since $\mathbf{F}$ is nonsingular, recall the polar decomposition theorem

$$
\mathbf{F}=\mathbf{R U}
$$

where $\mathbf{R}$ is a proper orthogonal tensor and $\mathbf{U}$ is a positive definite symmetric tensor which can be calculated from $\mathbf{U}^{2}=\mathbf{F}^{T} \mathbf{F}$. In Naghdi and Vongsarnpigoon [5], the differential equation for the rotation tensor $\mathbf{R}$ is given for a constrained theory. In the context of the present development, it can be shown by a similar procedure that the differential equation for $\mathbf{R}$ is given by

$$
\mathbf{R}^{T} \mathbf{R}_{, \alpha}=\mathscr{A}_{\alpha},
$$

where

$$
\begin{aligned}
\mathscr{A}_{\alpha}= & -\mathscr{A}_{\alpha}^{T}=\frac{1}{2} \mathbf{U}^{-1}\left\{\mathbf{U}_{, \alpha} \mathbf{U}-\mathbf{U} \mathbf{U}_{, \alpha}+(\mathbf{U U})_{, \gamma}\left(\mathbf{A}_{\alpha} \otimes \mathbf{A}^{\gamma}\right)-\left(\mathbf{A}^{\gamma} \otimes \mathbf{A}_{\alpha}\right)(\mathbf{U U})_{, \gamma}\right. \\
& +\left(\mathbf{F}^{T} \mathbf{G}+\mathbf{G}^{T} \mathbf{F}-\mathbf{U} \mathbf{U}_{0} \mathbf{G}-{ }_{0} \mathbf{G}^{T} \mathbf{U} \mathbf{U}\right)\left(\mathbf{A}_{\alpha} \otimes \mathbf{D}^{3}\right) \\
& \left.-\left(\mathbf{D}^{3} \otimes \mathbf{A}_{\alpha}\right)\left(\mathbf{F}^{T} \mathbf{G}+\mathbf{G}^{T} \mathbf{F}-\mathbf{U} \mathbf{U}_{0} G-{ }_{0} \mathbf{G}^{T} \mathbf{U} \mathbf{U}\right)\right\} \mathbf{U}^{-1} .
\end{aligned}
$$

The integrability conditions can be obtained from the requirement that $\mathbf{R}_{, \alpha \beta}=\mathbf{R}_{, \beta \alpha}$.

For a deformation with $\mathbf{E}=\mathbf{O}(\varepsilon)$ and $\mathbf{E}_{, \alpha}=\mathbf{O}(\varepsilon)$, if terms of $O\left(\varepsilon^{2}\right)$ are neglected, (A8) can be written in the form 


$$
\begin{aligned}
\mathscr{A}_{\alpha}=-\mathscr{A}_{\alpha}^{T}= & \left\{\frac{1}{2}\left(\kappa_{\beta \alpha}+\kappa_{\alpha \beta}\right)-\gamma_{3 \alpha|| \beta}+D \gamma_{\beta \lambda} B_{\alpha}^{\lambda}-\gamma_{3 \beta} \frac{D_{, \alpha}}{D}\right. \\
& \left.+\frac{\gamma_{33}}{D} B_{\beta \alpha}-\frac{1}{2}\left(\kappa_{\beta \alpha}+\kappa_{\alpha \beta}\right) \frac{\gamma_{33}}{D^{2}}+\frac{1}{2}\left(\kappa_{\gamma \alpha}+\kappa_{\alpha \gamma}\right) \gamma_{\lambda \beta} A^{\lambda \gamma}\right\} \\
\left(\mathbf{D}^{\beta} \otimes \mathbf{D}^{3}-\mathbf{D}^{3} \otimes \mathbf{D}^{\beta}\right) & \\
& +\left\{\gamma_{\gamma \alpha|| \beta}-\gamma_{\beta \alpha|| \gamma}+\frac{\gamma_{3 \beta}}{D} B_{\gamma \alpha}-\frac{\gamma_{3 \gamma}}{D} B_{\beta \alpha}\right. \\
& \left.+\frac{1}{2}\left(\kappa_{\beta \alpha}+\kappa_{\alpha \beta}\right) \frac{\gamma_{3 \gamma}}{D^{2}}-\frac{1}{2}\left(\kappa_{\gamma \alpha}+\kappa_{\alpha \gamma}\right) \frac{\gamma_{3 \beta}}{D^{2}}\right\} \mathbf{D}^{\gamma} \otimes \mathbf{D}^{\beta} .
\end{aligned}
$$

Making use of the relationship between $\kappa_{\alpha \beta}$ and $\beta_{\alpha \beta}$, (A9) can be shown to be equivalent to

$$
\begin{aligned}
\mathscr{A}_{\alpha}= & \left.-\beta_{\alpha \beta}-\frac{1}{2}\left(\gamma_{3 \alpha|| \beta}-\gamma_{3 \beta|| \alpha}\right)+\gamma_{\beta \lambda} A^{\lambda \gamma} b_{\gamma \alpha}-\gamma_{3 \beta} \frac{D_{, \alpha}}{D^{2}}\right\} \\
& \cdot\left(\mathbf{A}^{\beta} \otimes \mathbf{A}_{3}-\mathbf{A}_{3} \otimes \mathbf{A}^{\beta}\right) \\
+ & \left\{\gamma_{\gamma \alpha|| \beta}-\gamma_{\beta \alpha|| \gamma}+\gamma_{3 \beta} \frac{b_{\gamma \alpha}}{D}-\gamma_{3 \gamma} \frac{b_{\beta \alpha}}{D}\right\} \mathbf{A}^{\gamma} \otimes \mathbf{A}^{\beta}
\end{aligned}
$$

With respect to (A7), we can state and prove a theorem and a corollary parallel to Theorem 3.1 and Corollary 3.1 of Naghdi and Vongsarnpigoon [5]. Consequently, corresponding to given $\mathbf{U U}$ and $\mathbf{F}^{T} \mathbf{G}$, the deformation of the Cosserat surface can be determined to within a rigid body motion. In addition, we can state the following theorems:

Theorem A1. Given $\mathbf{E}=\mathbf{O}(\varepsilon), \mathbf{E}_{, \alpha}=\mathbf{O}(\varepsilon)$ and $\beta_{\alpha \beta}=O(1)$, the rotation tensor $\mathbf{R}$ associated with the deformation is a finite rotation.

TheOREM A2. Given $\mathbf{E}=\mathbf{O}(\varepsilon), \mathbf{E}_{, \alpha}=\mathbf{O}(\varepsilon)$ and $\beta_{\alpha \beta}=O\left(\varepsilon^{1 / 2}\right)$, the rotation tensor $\mathbf{R}$ associated with the deformation is a moderate rotation with respect to $\varepsilon$.

THEOREM A3. Given $\mathbf{E}=\mathbf{O}(\varepsilon), \mathbf{E}_{, \alpha}=\mathbf{O}(\varepsilon)$ and $\beta_{\alpha \beta}=O(\varepsilon)$, the rotation tensor $\mathbf{R}$ associated with the deformation is an infinitesimal rotation with respect to $\varepsilon$.

Theorem A2 corresponds to Theorem 3.2 of Naghdi and Vongsarnpigoon [5]; and the proofs of all three theorems are parallel to that of Theorem 3.2 of Naghdi and Vongsarnpigoon [5].

\section{REFERENCES}

[1] P. M. Naghdi, The theory of shells and plates, in S. Flügge's Handbuch der Physik, Vol. VIa/2 (edited by C. Truesdell), Springer-Verlag, Berlin, 1972, 425-640

[2] P. M. Naghdi, Finite deformation of elastic rods and shells. Proc. IUTAM Symp. on Finite Elasticity (Bethlehem, PA, 1980, edited by D. E. Carlson and R. T. Shield), Martinus Nijhoff Publishers, Hague, Netherlands, 1982, 47-103

[3] J. Casey and P.,M. Naghdi, An invariant infinitesimal theory of motion superposed on a given motion, Arch. Rational Mech. Anal. 76, 355-390 (1981)

[4] P. M. Naghdi and L. Vongsarnpigoon, Small strain accompanied by moderate rotation, Arch. Rational Mech. Anal. 80, 263-294 (1982) 
[5] P. M. Naghdi and L. Vongsarnpigoon, $A$ theory of shells with small strain accompanied by moderate rotation, Arch. Rational Mech. Anal. 83, 245-283 (1983)

[6] L. P. Eisenhart, An introduction to differential geometry. Princeton University Press, Princeton, 1947

[7] B. O'Neill, Elementary differential geometry, Academic Press, New York, 1966

[8] P. M. Naghdi and R. P. Nordgren, On the nonlinear theory of elastic shells under the Kirchhoff hypothesis, Quart. Appl. Math. 21, 49-59, (1963)

[9] E. Reissner, On axisymmetrical deformation of thin shells of revolution, Prov. Symp. Appl. Math. 3, 27-52 (1950) 